\title{
Mobile Health como herramienta en la prevención del acoso escolar: caso de estudio educación básica superior
}

\author{
Mobile Health as a tool in the prevention of bullying: case study \\ higher basic education
}

1 Genesis Dayana Pinto Almeida

https://orcid.org/0000-0003-2647-1735

Universidad Técnica de Ambato, Carrera de Educación Básica, Tungurahua,

gpinto8327@uta.edu.ec

2 Raúl Yungán Yungán

https://orcid.org/0000-0003-0280-8540

Universidad Técnica de Ambato, Carrera de Educación Básica, Tungurahua,

ryungan@uta.edu.ec

3 Evelyn Paulina Rovalino Ortega $\quad$ (iD) https://orcid.org/0000-0003-2909-3514

Universidad Técnica de Ambato, Carrera de Educación Básica, Tungurahua,

erovalino0178@uta.edu.ec

4 Mirian Janeth Bastidas Carrasco $\quad$ iD https://orcid.org/0000-0002-7316-8483

Universidad Técnica de Ambato, Carrera de Educación Básica, Tungurahua,

\section{Artículo de Investigación Científica y Tecnológica}

Enviado: 24/12/2021

Revisado: 29/12/2021

Aceptado: $12 / 01 / 2022$

Publicado:08/03/2023

DOI: https://doi.org/10.33262/concienciadigital.v6i1.4.2021

Cítese. $\quad$ Pinto Almeida, G. D., Yungán Yungán, R., Rovalino Ortega, E. P., \& Bastidas Carrasco, M. J. (2023). Mobile Health como herramienta en la prevención del acoso escolar: caso de estudio educación básica superior . ConcienciaDigital, 6(1.4), 670-684. https://doi.org/10.33262/concienciadigital.v6i1.4.2021

CONCIENCIA DIGITAL, es una Revista Multidisciplinar, Trimestral, que se publicará en soporte electrónico tiene como misión contribuir a la formación de profesionales competentes con visión humanística y crítica que sean capaces de exponer sus resultados investigativos y científicos en la misma medida que se promueva mediante su intervención cambios positivos en la sociedad. https://concienciadigital.org _

La revista es editada por la Editorial Ciencia Digital (Editorial de prestigio registrada en la Cámara Ecuatoriana de Libro con No de Afiliación 663) www.celibro.org.ec 
Palabras claves:

Mobile Health, aplicación móvil, acoso escolar, autoestima, bullying
Keywords:

Mobile Health, mobile application, bullying, selfesteem, bullying
Resumen

Introducción. El siguiente trabajo abordó el uso de la tecnología móvil para identificar y contrarrestar el acoso escolar entre los alumnos que son acosados de manera constante y de diversos modos, pueden ser de forma verbal, física, psicológica y en la actualidad hasta virtual dada por redes sociales. Objetivo. Verificar mediante el uso tecnológico "Mobile Health" el acoso escolar e identificar la presencia de conflictos existentes entre docente - alumno y entre estudiantes, a más de lograr establecer una aplicación móvil con el fin de generar empatía que ayude a evitar y manifestar el acoso escolar tanto dentro como fuera del aula. Metodología. Para la investigación el tipo de metodología fue experimental - exploratoria con el objeto de analizar causas y en consecuencia el efecto que se da en los involucrados, de igual manera se usó el enfoque cuantitativo. Para la recolección de información se ejecutó la encuesta como técnica e instrumento el cuestionario estructurado enfocado a los estudiantes y una ficha de observación dirigido a docentes, de igual forma el método T.A.M. (Technology Acceptance Model). La población son los alumnos de noveno de Educación General Básica de la Unidad Educativa Juan León Mera "La Salle". Resultados. Para la comprobación de la hipótesis se utilizó Kolmogorov-Smirnov encontrado dentro del parámetro de comprobación. Los estudiantes aseguraron no sentirse intimidados por los docentes en un $69,2 \%$, de igual manera mencionaron en un $47 \%$ nunca haberse sentido ofendidos por sus compañeros, sin embargo, un $36,8 \%$ señalaron que ocasionalmente sí. Conclusión. Se concluyó que la aplicación AfriEndly fue bien recibida y aceptada tecnológicamente, comprobado mediante el Método T.A.M, que a pesar de ser pocos los alumnos que se sienten acosados la App aportó a 113 de 117 estudiantes que la descargaron en sus móviles.

\section{Abstract}

Introduction. The following paper addressed the use of mobile technology to identify and counter bullying among students who are constantly bullied and in various ways, they can be verbally, physically, psychologically and currently even virtually given by social networks. Objective. Verify through the technological use "Mobile health" bullying and identify the presence of conflicts 
between teacher - student and between students, in addition to establishing a mobile application, to generate empathy that helps prevent and manifest bullying both inside and outside the classroom. Methodology. For the research the type of methodology was experimental - exploratory to analyze causes and consequently the effect that occurs in those involved, in the same way the quantitative approach was used. For the collection of information, the survey was executed as a technique and instrument the structured questionnaire focused on students and an observation sheet aimed at teachers, as well as the T.A.M. method (Technology acceptance model). The population is the ninth-grade students of Basic General Education of the Juan León Mera La Salle Educational Unit. Results. For the test of the hypothesis, Kolmogorov-Smirnov found within the test parameter was used. Students said they were not intimidated by teachers in $69.2 \%$, In the same way, $47 \%$ mentioned never having been offended by their peers, however, $36.8 \%$ said that occasionally they did. Conclusion. It was concluded that the AfriEndly application was well received and technologically accepted, using the T.A.M, that despite being few students who feel harassed the App contributed to 113 of 117 students who downloaded it on their mobiles.

\section{Introducción}

\section{Mobile Health}

La tecnología móvil ha ido evolucionando con el tiempo y por ende el cambio se ha visto reflejado en el vivir de cada sujeto, algunos aseguran que ha sido en beneficio del mundo gracias a la comunicación a larga distancia y diversas facilidades que el móvil brinda, sin embargo, otros aseguran que la tecnología está consumiendo la vida de cada individuo, pues hoy en día existen más celulares que seres humanos. Molina (2021) en el artículo titulado "La importancia de conocer y escuchar a todos los implicados en el diseño y uso de las aplicaciones móviles de nutrición" presenta un enfoque competente acorde a la salud mediante la tecnología móvil ofreciendo información acerca de patrones alimenticios saludables. Además, resaltan brindar motivación conforme al cambio de rutina y comportamiento, todo ello en tal solo una aplicación.

Por lo tanto, el término Mobile Health según Teixeira (2021), comprende atención y praxis en la salud por medio de aplicaciones en dispositivos móviles, englobando una estrategia con el fin de facilitar esa atención. Aquellas estrategias incluyen facilidades 
para el usuario, tales como llamadas telefónicas desde cualquier lugar, mensajes de texto enviado al desarrollador de la app, la misma puede ofrecer recomendaciones y diferentes usos para beneficio del usuario. La salud móvil ha evidenciado apoyar a la toma de decisiones conforme a la telemedicina, mostrando no solo efectividad en la interacción del usuario con el paciente, si no la adopción de estilos de vida diferente (Morais, 2022).

Por ello, en la investigación de Ribeiro \& Cestari (2022) "benchmarking of mobile apps on heart failure", menciona que para facilitar el proceso de atención a las personas con IC destacan las tecnologías de mHealth, como las aplicaciones móviles (APP). En los últimos años, el número de APP se ha incrementado exponencialmente en las más variadas áreas y ha contribuido a incrementar la productividad y la calidad de la atención sanitaria, además de permitir el uso del conocimiento clínico más actualizado y apoyar la decisión clínica. elaboración de profesionales (2-3). Cuando se llevan a cabo en la atención de pacientes con IC, las APP contribuyen a la supervivencia y mejoran la calidad de vida.

Las plataformas tecnológicas, y en general, dentro de la medicina, Mobile Health ha resaltado como un beneficio dirigido a los pacientes, cuya metodología adherida a la rama de la medicina abarca el desarrollo de aplicaciones móviles con el propósito de otorgar salud electrónica. En la investigación de Garivia (2021) "ciber salud y salud móvil: adherencia al tratamiento en enfermedades crónicas" resalta que la salud móvil llega a ser una estrategia útil en diferentes áreas de la salud, por ejemplo en enfermería, farmacéutica, fisioterapia, nutrición, odontología, psicología e incluso en atención prehospitalaria, sin embargo el término Mobile Health es escaso en cuanto a definiciones e incluso en práctica, poco se ha escuchado o leído de la salud móvil como algo cotidiano dentro de cualquier área e inclusive de la medicina.

Es así como, la tecnología no solamente pretende dar beneficios en la salud, sino también en la educación y aquello se lo denomina Mobile Learning, cuyo aprendizaje es basado en aplicaciones móviles pretendiendo innovar la forma de aprender, pasando de una educación tradicional a una constructivista moderna. Cobos (2020) en el estudio "El Mobile Learning mediado con metodología PACIE para saberes constructivistas" asegura que el aprendizaje mediante la tecnología móvil impulsa al pedagogo a diseñar, planificar, desarrollar materiales dialógicos comunicativos que permitan transformar los procesos de enseñanza-aprendizaje por medio del móvil, es decir motivar a obtener experiencias cognitivas en cualquier momento y lugar.

En otras palabras, este tipo de metodología permite que el docente y el alumno mantengan una comunicación constante en cualquier momento del día, permitiendo una formación individualizada, a fin de que el pedagogo adapte el aprendizaje en base a las necesidades del estudiantado. De este modo Sousa (2021), destaca que Mobile Learning llega a influir de manera positiva en el aprendizaje del alumno, otorgando interés por lo que aprende, lo 
que conlleva a un resultado eficaz en la enseñanza adhiriendo un entorno virtual moderno y de esta forma permitiendo que el estudiante construya sus propios conocimientos, destrezas y habilidades digitales atrayendo su atención en cualquier lugar. Es decir, la modernidad ha llevado a los docentes a actualizarse de forma constante satisfaciendo las demandas conforme a la educación y formación tecnológica, puesto que mediante el internet se logra aprender cosas nuevas sin limitaciones conforme al tiempo y así mismo al lugar y aquellas aplicaciones móviles de innovación al aprendizaje posee un enfoque moderno (Rojos, 2021).

\section{Acoso escolar}

El acoso escolar consiste en un hostigamiento escolar, esta puede verse reflejado mediante el maltrato físico, verbal y psicológico ocasionado entre los estudiantes e incluso puede darse por parte de los profesores de manera reiterada a un largo o corto tiempo. Tepale (2021) en el artículo "Vivencias de acoso escolar en jóvenes: análisis y reflexión desde el psicoanálisis" asegura que mayormente los jóvenes que padecen de bullying son objeto de vulnerabilidad ante la sociedad, ya sea por el nivel socioeconómico, las profesiones o nivel de estudio de los padres, puede ser por ser sujeto migrante o ser diferente en cuestiones como en la personalidad, color de cabello, altura, edad, color de piel entre otros aspectos, e incluso son hostigados de manera continua gracias a su rendimiento académico, éste puede ser alta y denominarse como "nerd" o calificaciones bajas denominado como "vago".

La violencia según De Oliveira \& Franca (2022), llega a ser un fenómeno no visible, por lo general, la víctima suele padecer todo el acoso en silencio por miedo a sus abusadores, sin embargo, suelen darse a conocer señales que pretenden pedir ayuda a las personas que los rodea, como a la familia, amistades y docentes. Las señales pueden presentase en términos como: vos débil, y como consecuencia pueden mantener agachado la cabeza con la mirada al piso, silencio permanente, enfermedades inventadas con el fin de ausentarse en la escuela, bajo nivel en el desempeño académico, desgano de convivir por sentirse amenazado, por lo que pueden presentar baja autoestima, inseguridad, cuadros depresivos e ideas suicidas.

Pardo (2021) sugiere que el acoso escolar se presenta como una de las principales categorizaciones de la violencia escolar y se define por las acciones negativas, intencionales y repetitivas de los estudiantes en relación con los demás, que ocurren a partir de la desigualdad de poder entre el autor (es) y su víctima. Aquello, se trata de un problema social y de salud que se caracteriza por la emisión de conductas agresivas y antisociales en el entorno de las escuelas, en sus alrededores o en el camino casa-escuela, cuyo fenómeno, se manifiesta a través de agresiones físicas, psicológicas, sexuales y / o patrimoniales y puede involucrar a estudiantes, docentes, empleados, administrativos, directivos, familiares y residentes del contexto educativo, donde se ubica la escuela, por 
lo que la frecuente ocurrencia de esta violencia afecta negativamente la salud y la calidad de vida de toda la comunidad escolar (Lanne \& Luna, 2021).

Reisen (2021) resalta que el conocimiento de los pedagogos y las creencias sitúan sus estrategias de respuesta al acoso, lo que puede contribuir a una respuesta a tal violencia cese. Es así como identifican la existencia de tres creencias generales: primeramente, los estudiantes no se sentirían intimidados si se defendieran de la agresión; segundo, los alumnos no se sentirían intimidados si evitaran el contacto con los agresores; y tercero el acoso es un comportamiento normativo que ayuda a aprender las normas sociales. Almagro (2021) destaca que cuando el docente no interviene en el bullying, los estudiantes se sienten inseguros en la escuela y que de nada sirve pedir ayuda a sus profesores. Por ello, es importante conocer las creencias e ideologías de los pedagogos en correspondencia al bullying y su percepción de autoeficacia para enfrentar este problema con el fin de construir programas efectivos para combatir el bullying.

Por lo tanto, las instituciones educativas necesitan de docentes observadores con relación a su entorno escolar, que estén pendientes a las demandas y necesidades de sus estudiantes a más de lograr un aprendizaje significativo en un ambiente inclusivo y de practica de valores. Gallego (2021) en el estudio "El bullying en el marco de la escuela inclusiva: revisión sistemática" resaltan que un modelo que trabaje sin exclusiones para lograr atender en su totalidad a la diversidad del estudiantado, considerando al acoso escolar como un tópico relevante a ser tratado como un proceso de aprendizaje direccionado al respeto, confianza, felicidad, a una vida sin violencia, sin temores, en un ambiente educativo inclusivo y pacífico.

\section{Metodología}

El tipo de investigación fue experimental - exploratoria a razón de obtener las causas del por qué se da el acoso escolar y así mismo anteponer una aplicación móvil que contribuya a detectar tal violencia y a prevenirlo, a más de haber sido un estudio con una solución poco usual. El enfoque se dio por partes, primeramente, cualitativa, puesto que los datos obtenidos fueron gracias a la técnica: ficha de observación dirigida a los pedagogos que imparten clases a los estudiantes de noveno grado de E.G.B y cuantitativa debido a los datos obtenidos mediante una encuesta estructurada dirigido a los alumnos de noveno paralelos "A", "B" y "C" de E.G.B.

La técnica utilizada fue la observación para el desarrollo del instrumento denominado ficha de observación, la cual constó de una introducción en la que se detallaba la asignatura, el nombre del observador y la fecha, consecuente a los datos se estipuló una frecuencia del 1 al 5 siendo el más alto, a más de 10 indicadores relacionados al tópico de investigación, el cual contribuyó a identificar el acoso escolar del docente hacia los estudiantes. De igual manera, otra técnica utilizada fue la encuesta con su instrumento el 
cuestionario estructurado dirigido a los alumnos de noveno E.G.B, el cual constó inicialmente de 8 preguntas conformado por datos informativos excepto el nombre del estudiante, a continuación de 18 preguntas que contribuían a identificar la existencia de acoso entre alumnos y de igual forma se tomó en consideración la percepción del estudiante ante los docentes.

Para el desarrollo del estudio se usó la metodología A.D.D.I.E: Análisis; Diseño; Desarrollo; Implementación; y Evaluación.

Análisis: Se analizó como pre-test el cuestionario estructurado dirigido a los alumnos de noveno E.G.B. aplicado en horas de clases virtuales. De igual manera se empleó las fichas de observación dirigido a docentes de las diferentes asignaturas, con el fin de reconocer si los pedagogos acosan a los estudiantes en base a su comportamiento. Para ello, la herramienta Mobincube fue un factor relevante a considerarse dentro del estudio, puesto que ayudaba a desarrollar una app gratuita eficaz y factible según diversos usuarios de plataformas en YouTube.

Diseño: El fin de la interfaz de Mobincube es interactuar el accionar a otros sistemas de forma fácil conforme a su uso y efectivo entre sujeto-ordenador, aquello concibió relevancia en generar un interfaz no solo factible al uso, sino también óptimo a cualquier individuo de diferente edad. Para ello, en el desarrollo de la app se consideró un "Menú" con 4 botones importantes, tales como el detector de acoso, el agresor, la víctima, las sugerencias que la app proporciona y los créditos.

Desarrollo: Mobincube nos ofreció un software gratuito y fácil de usar, gracias a que no es obligatorio insertar códigos y cuyo desarrollo fue con programación por bloques, accediendo a interfaces con imágenes interactivas, íconos, texto y tablas.

\section{Figura 1}

Menú Principal detector de acoso

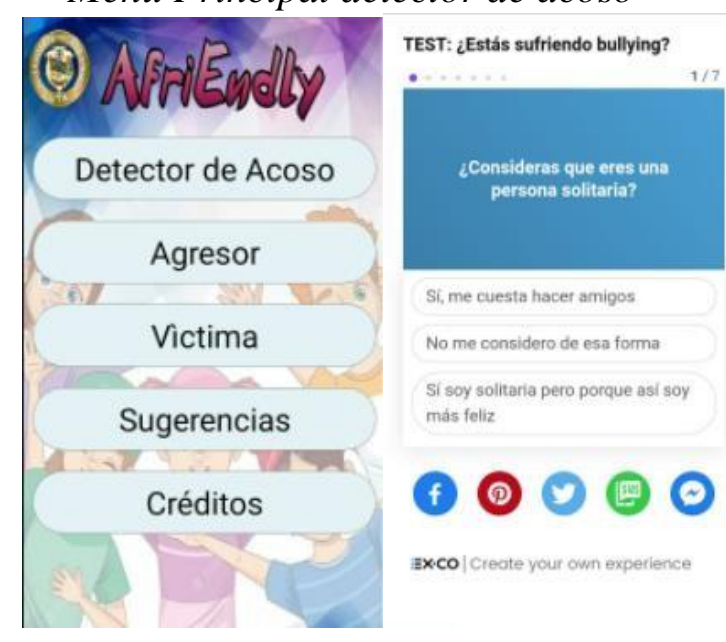

Elaborado por: Pinto 
Detector de Acoso: el menú principal consta de un botón denominado "Detector de Acoso", dirigiendo a una página Web "Test", el cual consigan siete preguntas que el usuario puede responder con el fin de conseguir al final de la encuesta un resultado, es decir la página le ayudará a conocer si sufre o no de bullying.

Figura 2

Menú Principal detector de acoso
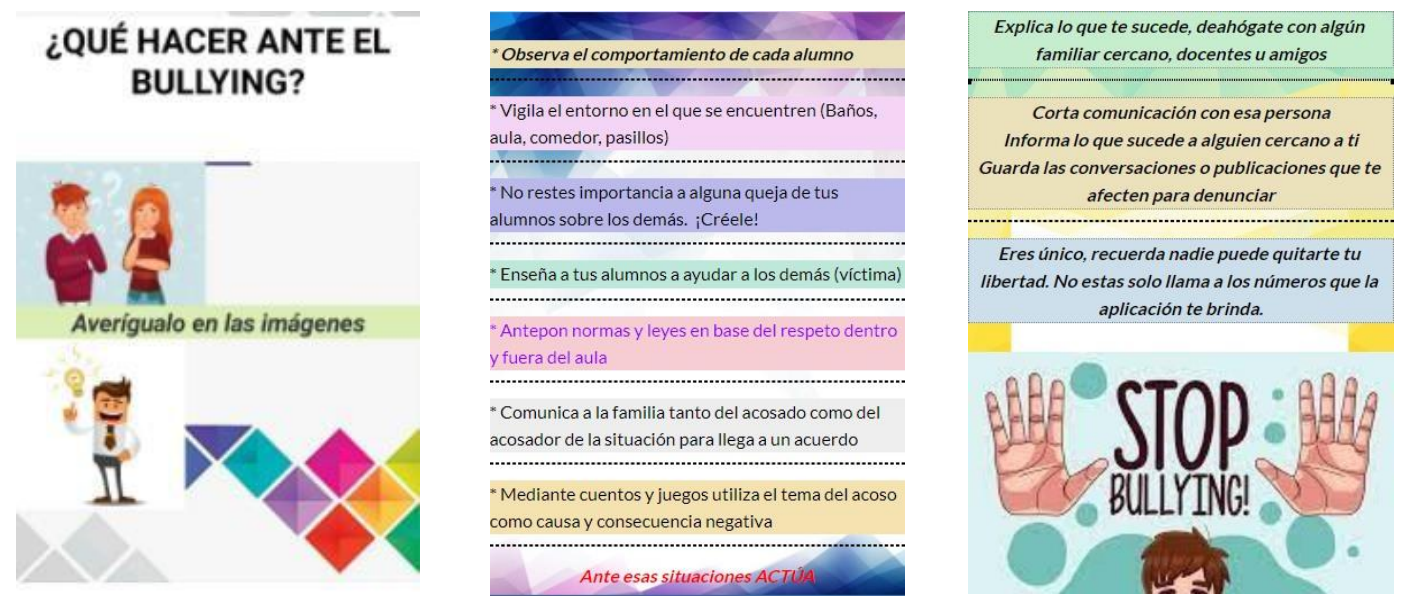

Elaborado por: Pinto

Sugerencias: la opción denominada "Recomendaciones" dirige a una nueva pantalla en la cual nos muestra dos imágenes, la de los jóvenes guía a una pantalla de sugerencias que los estudiantes pueden hacer si sufren de acoso, mientras que la imagen del docente envía a una pantalla donde sugiere que hacer ante observar acoso dentro de su aula como tutor.

Implementación: Para la adecuada instalación de la app los usuarios debían contar con una conexión de internet y acceder a Play Store, para ello se debió poseer celulares con sistema Android. Además, se consideró que para realizar alguna llamada es necesario tener saldo en el celular a excepción de la opción emergencia que consta en la app.

Evaluación: Se consideró fundamental el desarrollo de la metodología T.A.M "Technology Acceptance Model", con el objeto de determinar si la aplicación fue rechazada o aceptada dentro de la población de estudio, con el propósito de indagar el impacto que la tecnología brinda a la sociedad y como la población percibe tal utilidad.

\section{Resultados}

1. ¿Con que frecuencia usted se ha sentido intimidado u ofendido por alguno de sus docentes provocando incomodidad en las horas clase? 
Tabla 1

Intimidación docente

\begin{tabular}{lcc}
\hline Items & Frecuencia & Porcentaje \\
\hline Nunca & 81 & 69,2 \\
Raramente & 27 & 23,1 \\
Ocasionalmente & 7 & 6,0 \\
Frecuentemente & 1 &, 9 \\
Muy frecuentemente & 1 &, 9 \\
Total & 117 & 100,0 \\
\hline
\end{tabular}

Elaborado por: Pinto.

Fuente: Encuesta aplicada a los estudiantes de noveno E.G.B "La Salle"

Figura 3

Intimidación

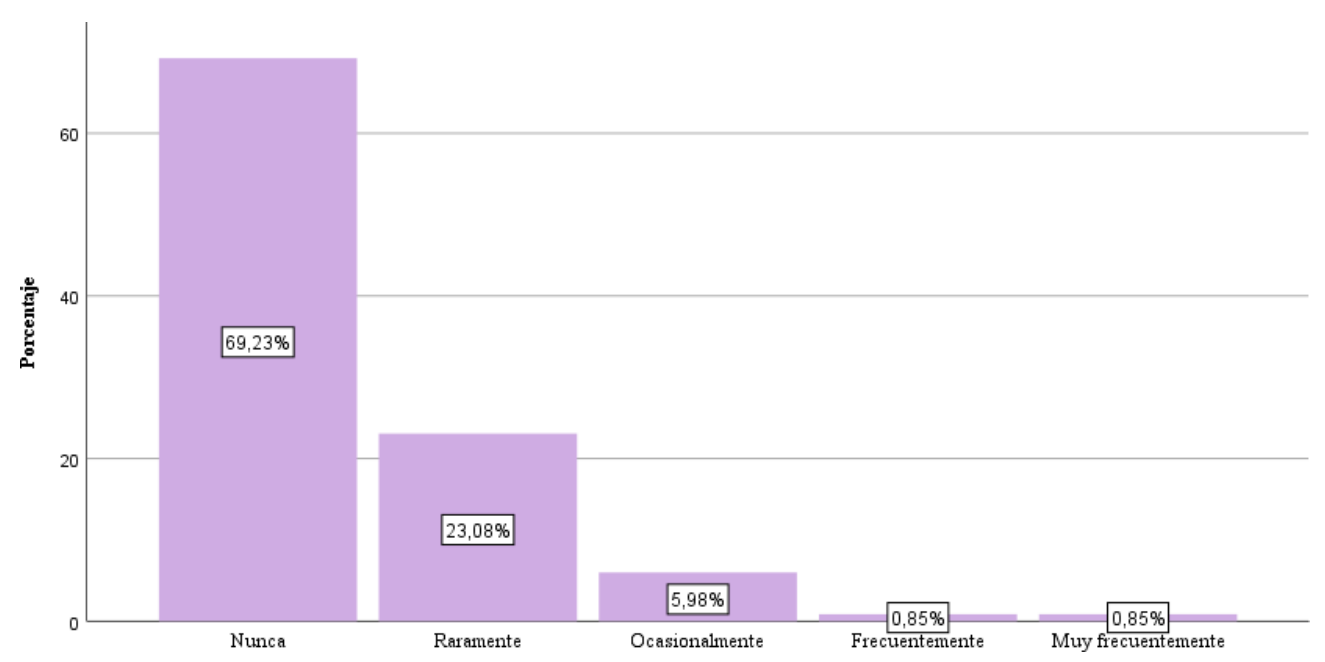

Elaborado por: Pinto.

Fuente: Encuesta aplicada a los estudiantes de noveno E.G.B "La Salle"

De un total de 117 estudiantes correspondientes al 100\% entre la muestra, 81 alumnos que representa el 69,23\% nunca se han sentido intimidado u ofendido por algún docente, 27 estudiantes que representan el 23,08\% raramente, el 5,98\% ocasionalmente, el 0,85\% frecuentemente consignado por 1 alumno y el $0,85 \%$ muy frecuentemente constado por 1 escolar. Lo que permite reconocer dentro de la investigación que la mayoría del estudiantado de noveno E.G.B. no se han sentido intimidados u ofendidos por alguno de sus docentes lo que consecuentemente no ha provocado incomodidad en las horas de clase.

2. ¿Con qué frecuencia usted se ha sentido ofendido por algún comentario hecho por sus compañeros, ya sea por medio de redes sociales o en persona? 
Tabla 2

Comentarios ofensivos

\begin{tabular}{lcc}
\hline İtems & Frecuencia & Porcentaje \\
\hline Nunca & 55 & 47,0 \\
Raramente & 43 & 36,8 \\
Ocasionalmente & 12 & 10,3 \\
Frecuentemente & 6 & 5,1 \\
Muy frecuentemente & 1 & 0,9 \\
Total & 117 & 100,0 \\
\hline
\end{tabular}

Elaborado por: Pinto.

Fuente: Encuesta aplicada a los estudiantes de noveno E.G.B "La Salle"

Figura 4

Comentarios ofensivos

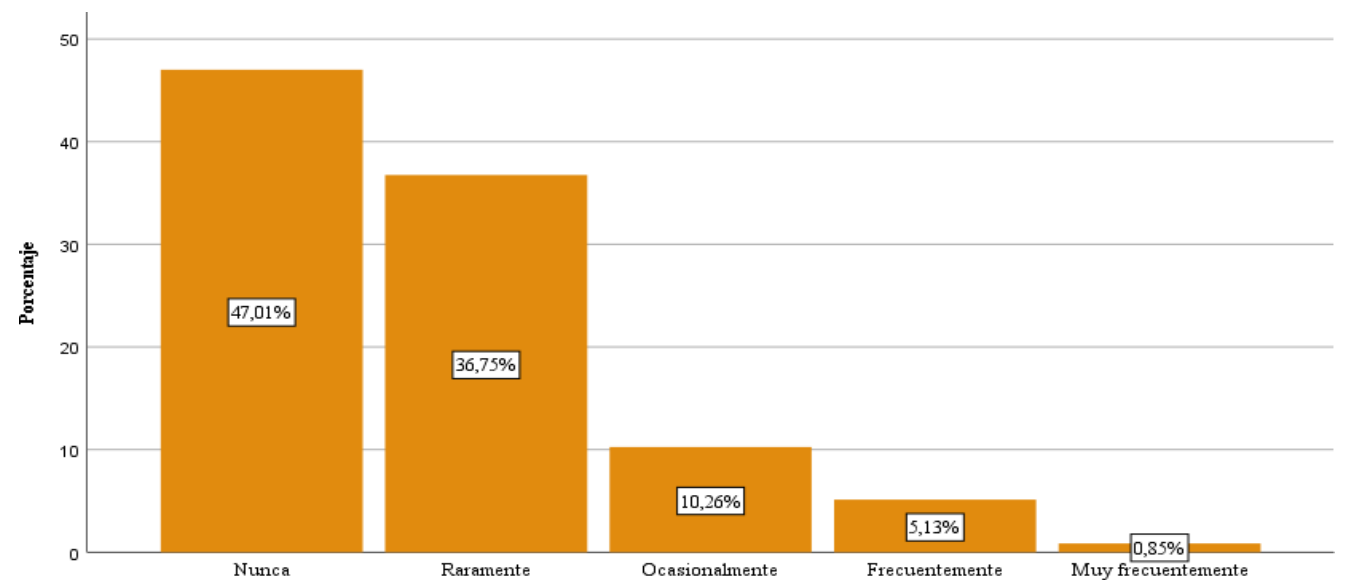

Elaborado por: Pinto.

Fuente: Encuesta aplicada a los estudiantes de noveno E.G.B "La Salle"

De un total de 117 estudiantes correspondientes al 100\% entre de la muestra, el 47,01\% equivalente a 55 alumnos nunca se han se han sentido ofendidos por algún comentario, el $36,75 \%$ raramente, el 10,26\% ocasionalmente, el 5,13\% frecuentemente consignado por 6 alumnos y el $0,85 \%$ muy frecuentemente constado por 1 escolar. Lo que permite reconocer dentro de la investigación que la mayoría del estudiantado de noveno E.G.B. se han sentido ofendidos por algún comentario hecho por sus compañeros, ya sea por medio de redes sociales o en persona, sin embargo 43 de ellos señalaron que raramente se han sentido disgustados por algún comentario.

\section{3. ¿Ha escuchado el término Mobile Health?}

Tabla 3

Término Mobile Health

\begin{tabular}{lcc}
\hline & Frecuencia & Porcentaje \\
\hline $\mathrm{Si}$ & 7 & 6,0 \\
No & 110 & 94,0 \\
Total & 117 & 100,0 \\
\hline
\end{tabular}

Elaborado por: Pinto.

Fuente: Encuesta aplicada a los estudiantes de noveno E.G.B "La Salle" 


\section{Figura 5}

Término Mobile Health

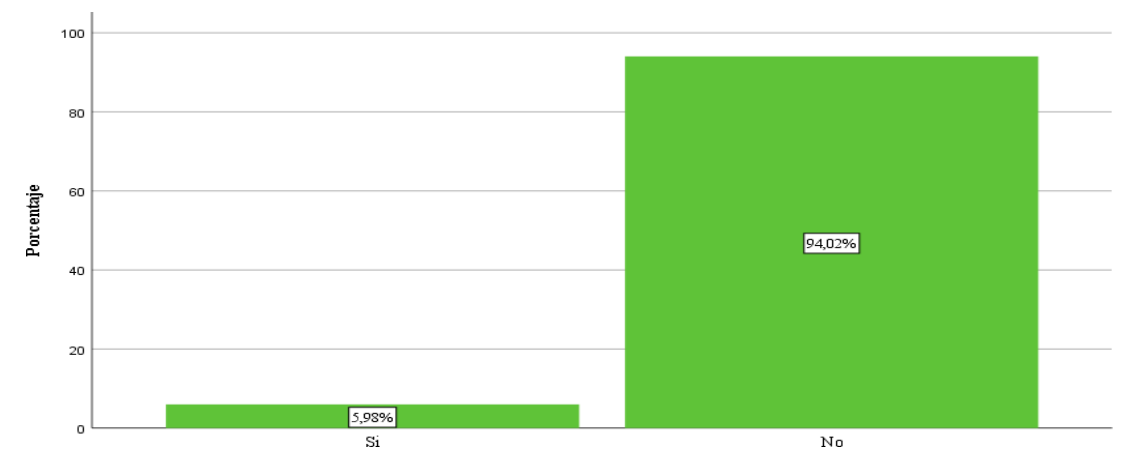

Elaborado por: Pinto.

Fuente: Encuesta aplicada a los estudiantes de noveno E.G.B "La Salle"

De un total de 117 estudiantes correspondientes al 100\% entre de la muestra, el 94,02\% equivalente a 110 alumnos resaltan que nunca han escuchado el término salud móvil y el 5,98\% señalaron que sí. Lo que permite reconocer dentro de la investigación que la mayoría del estudiantado de noveno E.G.B. nunca han escuchado el término Mobile Health.

4. ¿Conoce usted sobre alguna aplicación que detecte el acoso escolar o identifique el Bullying?

Tabla 4

Aplicación que detecte el acoso escolar

\begin{tabular}{lcc}
\hline & Frecuencia & Porcentaje \\
\hline $\mathrm{Si}$ & 8 & 6,8 \\
No & 109 & 93,2 \\
Total & 117 & 100,0 \\
\hline
\end{tabular}

Elaborado por: Pinto.

Fuente: Encuesta aplicada a los estudiantes de noveno E.G.B "La Salle"

\section{Figura 6}

Aplicación que detecte el acoso escolar

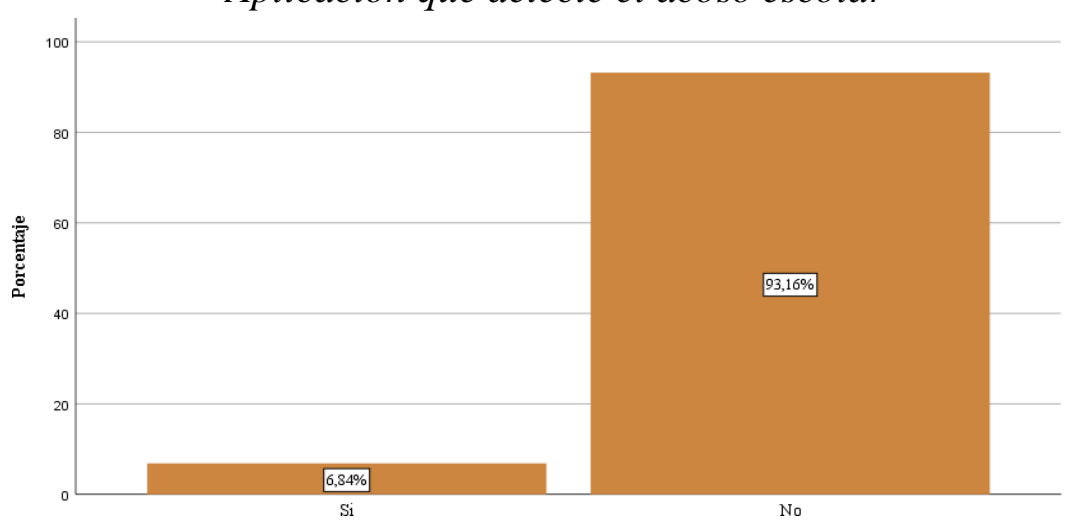

Elaborado por: Pinto.

Fuente: Encuesta aplicada a los estudiantes de noveno E.G.B "La Salle" 
De un total de 117 estudiantes correspondientes al 100\% entre de la muestra, el 93,16\% equivalente a 109 alumnos resaltan que no conocen ninguna aplicación que detecte el acoso y el 6,84\% señalaron que sí. Lo que permite reconocer dentro de la investigación que la mayoría del estudiantado de noveno E.G.B. no tienen conocimiento sobre alguna aplicación que detecte el acoso escolar o identifique el bullying.

\section{Verificación de Hipótesis}

La experimentación se desarrolló en la Unidad Educativa "La Salle" con una duración de seis semanas en las aulas de noveno grado de Educación General Básica, observadas en diferentes horarios y en diversas asignaturas, el mecanismo utilizado fue la app en los dispositivos móviles de los alumnos que conforman los tres cursos investigados. A sí mismo, en el estudio se contrastó una limitante al momento de la descarga de la aplicación, puesto que el único operativo en el que se logra obtener la app es en el sistema Android, más no en el sistema IOS.

Para la comprobación de la hipótesis se consideró el estadístico Kolmogorov-Smirnov o $\mathrm{K}$ - S, por medio de las dos preguntas distintivas a la investigación manifestadas en el experimento como tal. Las preguntas son: en relación con la Variable Independiente "Mobile Health" ¿Ha escuchado el término Mobile Health? y en la Variable Dependiente “Acoso Escolar” ¿Con qué frecuencia usted se ha sentido ofendido por algún comentario hecho por sus compañeros?

Tabla 5

Prueba de Kolmogorov-Smirnov para una muestra

\begin{tabular}{|c|c|c|c|}
\hline & & $\begin{array}{l}\text { Ha escuchado } \\
\text { el término } \\
\text { Mobile Health }\end{array}$ & $\begin{array}{l}\text { Con qué frecuencia usted se ha sentido } \\
\text { ofendido por algún comentario hecho por } \\
\text { sus compañeros, ya sea por medio de } \\
\text { redes sociales o en persona }\end{array}$ \\
\hline $\mathrm{N}$ & & 117 & 117 \\
\hline \multirow[t]{2}{*}{ Parámetros normales $s^{\mathrm{a}, \mathrm{b}}$} & Media & 1,94 & 1,76 \\
\hline & Desviación típic & 238 & 897 \\
\hline \multirow[t]{3}{*}{ Diferencias más extremas } & Absoluta &, 539 & 272 \\
\hline & Positiva & ,401 & ,272 \\
\hline & Negativa &,- 539 &,- 198 \\
\hline \multirow{2}{*}{$\begin{array}{l}\mathrm{Z} \text { de Kolmogorov-Smirnov } \\
\text { Sig. asintót. (bilateral) }\end{array}$} & & 5,834 & 2,941 \\
\hline & &, 000 & ,000 \\
\hline \multicolumn{4}{|c|}{$\begin{array}{l}\text { a. La distribución de contraste es la Normal. } \\
\text { b. Se han calculado a partir de los datos. }\end{array}$} \\
\hline
\end{tabular}

Elaborado por: Pinto. Fuente: Herramienta SPSS

\section{Conclusiones}

- El bullying es un factor relevante para observar dentro de las aulas, ya que las consecuencias de la violencia causan en los estudiantes agresiones físicas, 
verbales, psicológicas y actualmente la cibernética. Estas manifestaciones son graves, puesto que pueden provocar daños emocionales o psicológicos, físicos, y como consecuencia el estrés, dolores de barriga e incluso de la cabeza, pesadillas constantes, bajo rendimiento escolar, exclusión en el aula, entre otros. Por lo general, la víctima es sometida a diversas formas de violencia como: hostigamiento, manipulación social, golpes, insultos, apodos, entre muchas más situaciones desagradables por parte de los agresores. A consecuencia, las víctimas padecen en silencio deteriorando su autoestima y energía.

- Los estudiantes de los tres paralelos de noveno de Educación General Básica no padecen de acoso por parte de los docentes que imparten clases durante el periodo académico, sin embargo, se logró identificar que ocasionalmente la mayoría del estudiantado se sienten intimidados por sus compañeros.

- La tecnología hoy en día es usada no solo para interacción, sino también para recomendaciones de diversos tópicos que las aplicaciones suelen otorgar. Es así como, la aplicación desarrollada fue a fin de crear una guía de ayuda para la víctima, recomendando diversas vías de auxilio como números telefónicos e incluso fue una guía para el docente al conocer las diferentes acciones que puede tomar para evitar el acoso y fomentar la empatía.

\section{Referencias Bibliográficas}

Almagro, A. B. (2021). The Voice of Silence: Qualitative Evaluation of Bullying Practices in People with Intellectual Disability. Rev. bras. educ.

Cobos, V. P. (2020). Mobile-Learning mediated with PACIE methodology. Filosofía, tecnología e innovación en la educación.

De Oliveira, M. S. \& Franca, A. P. (2022). Violência contra crianças e adolescentes e pandemia - Contexto e possibilidades para profissionais da educação. Esc. Anna. Nery.

Gallego, M. R. (2021). Bullying in the context of inclusive education: asystematic review. Páginas de Educación.

Garivia, M. H. (2021). eHealth y mHealth: adherencia al tratamiento en enfermedades crónicas. Revista de la Facultad de Medicina.

Lanne Muniz, G. \& Luna, A. (2021). Crenças, autoeficácia e estratégias de professoresdiante do bullying. Scielo.

Molina, R. M. (2021). La importancia de conocer y escuchar a todos los implicados en el diseño y uso de las aplicaciones móviles de nutrición. Dando a conocer el Gran 
GApp. Nutrición Hospitalaria.

Morais, C. M. (2022). Mobile pre-hospital care reorganization during the COVID-19 pandemic: experience report. Rev. Bras. Enfermería.

Pardo, J. R. (2021). Los terceros en el bullying: ¿testigos o cómplices? Revista de la Asociación Española de Neuropsiquiatría.

Reisen, F. M. (2021). Associação entre capital social e bullying em adolescentes de 15 a 19 anos: relações entre o ambiente escolar e social. Ciênc. saúde coletiva.

Ribeiro, V. F. \& Cestari, R. S. (2022). Benchmarking of mobile apps on heart failure. Rev. Bras. Enfermería.

Rojos, M. S.-C. (2021). Plataforma Móvil Basada en la Nube para Mejorar la Experiencia de Aprendizaje en la Anatomía Humana. International Journal of Morphology.

Sousa, A. L. (2021). Mobile learning in teaching radiology in times of social isolation. Assoc. Med. Bras.

Tepale, S. Z. (2021). Bullying at School among Youth: Psychoanalytical Analysis and Reflection. Revista mexicana de investigación educativa.

Teixeira Andreotti, J. R. (2021). Detect-S: an mHealth application to assist health professionals to identify suicide risk in hospitalized patients. Trends Psychiatry Psychother.

\section{U Ciencia}


El artículo que se publica es de exclusiva responsabilidad de los autores y no necesariamente reflejan el pensamiento de la Revista Conciencia Digital.

\section{\Ciencia}

El artículo queda en propiedad de la revista y, por tanto, su publicación parcial y/o total en otro medio tiene que ser autorizado por el director de la Revista Conciencia Digital.
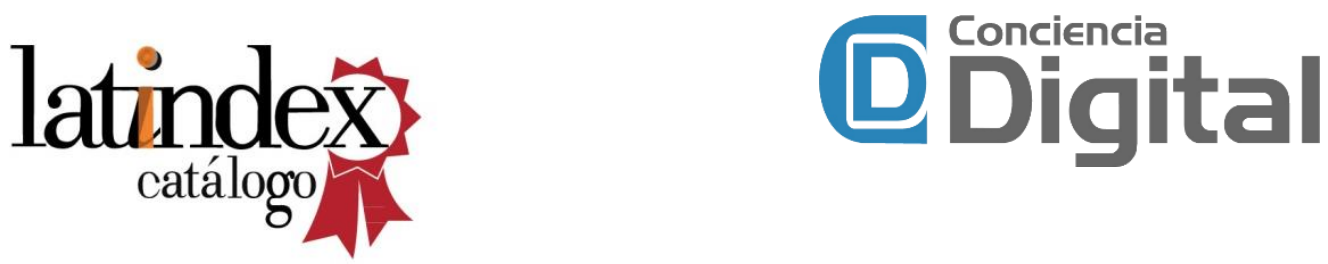

Indexaciones

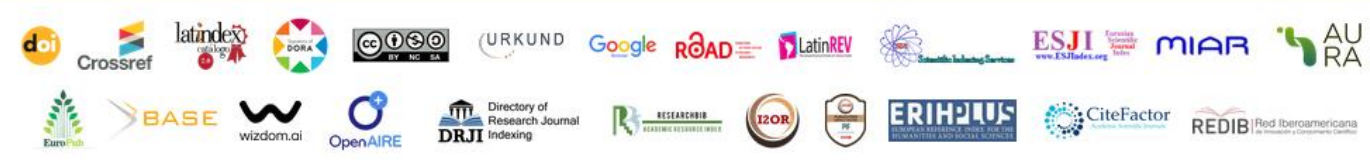

